Philosophie ANTIQUE

\section{Philosophie antique}

Problèmes, Renaissances, Usages

$18 \mid 2018$

L'athéisme antique

\title{
Sylvain Roux, L'être et le substrat. Essai sur Plotin et la métaphysique
}

\author{
Laurent Lavaud
}

\section{(2) OpenEdition}

\section{Journals}

Édition électronique

URL : https://journals.openedition.org/philosant/1124

DOI : 10.4000/philosant.1124

ISSN : 2648-2789

\section{Éditeur}

Éditions Vrin

\section{Édition imprimée}

Date de publication : 1 novembre 2018

Pagination : 294-297

ISBN : 978-2-7574-2372-1

ISSN : $1634-4561$

Référence électronique

Laurent Lavaud, «Sylvain noux, L'être et le substrat. Essai sur Plotin et la métaphysique », Philosophie antique [En ligne], 18| 2018, mis en ligne le 01 novembre 2018, consulté le 03 décembre 2022. URL http://journals.openedition.org/philosant/1124; DOI : https://doi.org/10.4000/philosant.1124

\section{c) (1) (9)}

Creative Commons - Attribution - Pas d'Utilisation Commerciale - Pas de Modification 4.0 International - CC BY-NC-ND 4.0

https://creativecommons.org/licenses/by-nc-nd/4.0/ 
à deux reprises contre Aristote « l'argument du simple ». Cet usage pourrait sembler paradoxal puisque Aristote lui-même ne rejette pas la simplicité du Premier moteur et la juge compatible avec sa causalité principielle. La suite de l'enquête de S. R., consacrée à d'autres traités de Plotin - Traité 7 (V 4) et Traité 30 (III 8) - et à d'autres textes de la Métaphysique d'Aristote, aboutit à mettre au jour deux conceptions distinctes de la simplicité chez les deux auteurs.

Elisa Coda explore la manière dont Thémistius prend en charge le problème philosophique de la nature du mouvement céleste et celui, exégétique, de la compatibilité des explications du De Caelo avec celles de la Physique et de la Métaphysique sur ce point. En revenant sur le texte du traité $\mathrm{Du}$ Ciel d'Aristote et sur les interprétations d'Alexandre et de Simplicius, puis en entrant dans le détail de la paraphrase par Thémistius de $D e$ Caelo II, 1 et II, 12 et de Métaphysique $\Lambda$, E. C. met en évidence que pour Thémistius les textes d'Aristote forment une explication cohérente du mouvement du Ciel conçu comme animé et mû par son désir d'imiter l'immobilité bienheureuse de la Première cause séparée. Cette interprétation, largement tributaire de celle d'Alexandre, a influencé la tradition arabe et notamment Averroès.

Enfin, Pantelis Golitsis montre que l'on trouve dans les commentaires du byzantin Michel d'Éphèse une approche néoplatonicienne caractéristique faisant de la Métaphysique une œuvre dirigée vers l'établissement du Dieu d'Aristote, premier principe, « substance éternelle, immobile et séparée des choses sensibles », pure Forme, pur Acte mais aussi, contre la doctrine péripatéticienne orthodoxe d'Alexandre, cause efficiente et père du cosmos. P. G. remonte aux origines de cette conception et offre un parcours général de la réception du Dieu d'Aristote dans le néoplatonisme : alors que Plotin et Proclus adoptent une attitude polémique, Ammonius opère une platonisation faible du Dieu d'Aristote en concevant cette intelligence comme cause finale et efficiente de l'univers et Simplicius, une platonisation beaucoup plus puissante en créditant Aristote de l'admission d'un premier principe supérieur à l'intelligence, l'Un-Bien. P. G. montre qu'Eustrate, métropolite de Nicée, fait sienne la conception de Simplicius tandis que Michel d'Éphèse s'en tient à celle d'Ammonius.

On soulignera pour finir la très grande qualité scientifique des contributions réunies dans ce volume. Cet ouvrage collectif relève le double défi d'offrir une remarquable synthèse sur la réception de la théologie d'Aristote, couvrant une ample période allant des successeurs immédiats du Stagirite jusqu'aux platoniciens byzantins, et d'apporter un éclairage inédit sur nombre de ses aspects, faisant apparaître dans toute sa diversité l'histoire des réappropriations et reformulations successives de ses concepts. Une riche bibliographie et trois index - noms anciens et médiévaux, noms modernes et contemporains, lieux - viennent utilement compléter ce volume, appelé à devenir une référence importante et un outil essentiel pour tout chercheur qui s'intéresse aux multiples devenirs de l'héritage aristotélicien.

FARAh Cherif Zahar

Université Paris 8, LLCP/Centre Léon Robin.

Sylvain Roux, L'être et le substrat. Essai sur Plotin et la métaphysique, Paris, Librairie Philosophique J. Vrin, 2017 (Histoire des doctrines de l'Antiquité classique), 295 p., ISBN 978-2-7116-2665-6.

L'ambition de ce livre est de définir le projet métaphysique plotinien en prenant pour fil directeur la question de la (non-) articulation entre l'être et le substrat. Selon l'auteur, la métaphysique de Plotin se situe, sur cette question, en rupture avec celle 
d'Aristote, qui, dans son analyse de l'ousia, ne parvient pas à dissocier l'essentialité de la substratité : dans les Catégories, comme dans la Métaphysique, la substance assume ultimement la fonction d'bupokeimenon, et, comme le note S. Roux, chez Aristote, « le projet métaphysique renvoie bien à un modèle qui est celui du substrat » (p. 45). Par contraste, Plotin cherche à privilégier un modèle métaphysique alternatif. Pour le montrer, l'auteur procède en trois temps. Il reprend tout d'abord la double critique quadresse Plotin, dans le Traité 42 (VI, 1) aux approches aristotélicienne et stoïcienne de la connexion entre la substance et le substrat. Puis il expose l'analyse plotinienne de la matière à travers l'examen des thèses présentées dans les Traités 12 (II, 4) et 44 (VI, 3). $S$. Roux entend démontrer que, même dans les passages consacrés à la matière intelligible où la notion d'hupokeimenon demeure prégnante, Plotin procède à un « réaménagement significatif » (p. 266) : si, d'un côté, le sens réel de l'ousia doit être réservé aux substances intelligibles, alors que les substances sensibles ne sont dites telles que par homonymie, à rebours, seules les réalités sensibles peuvent être dites au sens fort substrat, alors que les intelligibles ne sont désignées ainsi que selon l'homonymie. Enfin, dans une troisième partie, qui est sans doute la plus originale, $S$. Roux met en avant deux paradigmes métaphysiques alternatifs à celui de la substratité, qu'il baptise la « double inclusion » et la « coexistence ». Le premier paradigme met en jeu les grands genres intelligibles de l'être. Il a pour finalité d'établir la parfaite égalité ontologique entre l'ousia et les autres genres : l'idée par exemple que le mouvement est dans l'être doit être équilibrée par la relation réciproque selon laquelle l'être est dans le mouvement. Par cette réciprocité de la relation d'inclusion, Plotin subvertit la fonction de substrat de l'ousia intelligible et interdit de cantonner le mouvement (et les autres genres de l'être) au rôle ontologique dérivé d'accident de la substance. Le second paradigme, celui de la coexistence, s'applique en priorité à la relation entre l'intellection et l'intelligible, telle qu'elle est mise en scène dans le Traité 38 (VI, 7) : l'ousia et la pensée existent de façon égale et solidaire, sans qu'un terme jouisse d'une quelconque préséance sur l'autre, ce qui, une nouvelle fois, dissout la fonction substrative de la substance intelligible. D'après $S$. Roux, Plotin réaliserait par ailleurs, dans le Traité 39 (VI, 8) le transfert de ce modèle de la coexistence au niveau de l'Un : il servirait désormais à articuler l'un à l'autre le (quasi) être de l'Un et son (quasi) acte, à savoir la volonté. Cette articulation aurait pour but de dire la liberté pure de l'Un, capable de se vouloir et de se produire soi-même.

Pour mener à bien son enquête sur la « désubstratification » de l'ousia chez Plotin, S. Roux fait le choix radical de réduire à la portion congrue le débat avec la littérature secondaire - très abondante sur un tel sujet. Les commentateurs qui ont exploré cette question, tels par exemple Chiaradonna, Narbonne, Strange, Wurm ou, selon une problématique historique plus large, de Libera, sont certes cités, mais leurs thèses ne donnent pas lieu à une discussion critique très développée. Ce choix permet à l'auteur de concentrer toute son attention exégétique sur les textes mêmes de Plotin examinés avec patience et minutie, et dans un style à la fois toujours clair et soucieux de restituer scrupuleusement les complexités et les subtilités de la métaphysique de l'intelligible propre à Plotin. On peut ainsi lire ce livre comme un véritable précis d'ontologie plotinienne, qui recense tous les textes décisifs sur l'ousia.

Par ailleurs, S. Roux choisit aussi de limiter la mise en perspective historique de la métaphysique plotinienne à une confrontation directe avec Aristote et le stoïcisme. On peut suggérer cependant que sur le sujet précis du rapport entre l'ousia et le substrat, un examen attentif de certaines thèses des philosophes péripatéticiens plus proches de Plotin eût été fort instructif. Je pense par exemple au débat sur l'interprétation du sens de l'ousia, qui a opposé la lecture « substrative » de Boéthos de Sidon à celle, 
« essentialiste », d'Alexandre d'Aphrodise, dont on sait qu'il fut abondamment lu et commenté par Plotin.

Il est certain, en tout cas, que les thèses défendues par $S$. Roux concernant le mode singulier de la constitution plotinienne de la métaphysique suscitent la réflexion et invitent au débat. Je souhaiterais y apporter ma contribution à travers trois remarques. La première concerne l'idée, défendue avec vigueur, selon laquelle tout l'effort spéculatif de Plotin relativement à l'ousia intelligible tend à résister à la tendance substrative inhérente à la métaphysique d'Aristote. Ce point, dans la plupart des textes plotiniens consacrés à l'ousia intelligible, ne souffre pas de contestation. Il me semble cependant que la résurgence sporadique du vocabulaire du substrat pour caractériser la réalité « là-bas » n’épuise pas sa signification dans un simple et paisible « changement de sens » (p. 266) de l'hupokeimenon lorsqu'il se trouve transposé dans l'intelligible. Cette résurgence, particulièrement spectaculaire lorsque Plotin tente de donner une signification métaphysique à l'idée de matière intelligible, est plutôt selon moi le symptôme de l'extrême difficulté du projet plotinien d'arracher la métaphysique de l'ousia à sa figure substrative. Tout se passe comme si, dans certains passages des traités, l'écriture philosophique de Plotin était comme hantée par ce modèle aristotélicien de l'articulation entre un support et ses attributs, et qu'il lui fallait faire preuve d'un effort et d'une vigilance toujours repris pour parvenir à y échapper.

Ma deuxième remarque est relative au modèle de la double inclusion mis en œuvre en particulier dans le Traité 43. S. Roux souligne avec beaucoup de pertinence que la réciprocité de l'immanence (le fait par exemple que l'être soit tout autant dans le mouvement que le mouvement dans l'être) constitue une radicale subversion du paradigme de la substratité de l'ousia. Cette subversion doit cependant à mon sens être resituée dans le cadre d'une réduction hénologique de la théorie platonicienne des grands genres : la combinaison des grands genres entre eux constitue une ousia première, universelle, qui doit ensuite se déployer dynamiquement dans des genres et des espèces plus déterminés (j’ai défendu cette idée dans un article intitulé « The Primary Substance in Plotinus' Metaphysics : A Little-Known Concept », Phronesis, 59, 2014, p. 369-384). Par ailleurs, soulignons que ce modèle de la double inclusion aura une postérité décisive dans la réflexion trinitaire, en particulier chez Marius Victorinus et saint Augustin : l'égalité ontologique entre les genres devient chez ces auteurs un instrument conceptuel déterminant pour penser, contre l'arianisme, l'égalité entre les Personnes divines.

Enfin, revenons au modèle que $S$. Roux appelle de la « coexistence ». Dans le Traité 38, la sunhupostasis permet d'articuler, au niveau de l'Intellect, l'ousia intelligible et son acte propre, l'intellection. S. Roux évoque en passant, mais sans y souscrire totalement (p. 232), l'interprétation de P. Hadot, selon laquelle cette expression de sunhupostasis pourrait être restituée par le terme de « co-production ». Une telle traduction cependant a le mérite d'intégrer l'ontologie plotinienne dans une perspective dynamique et génétique (ce que rend moins sensible la simple idée de « coexistence ») : l'ousia et son acte intellectif «se font exister réciproquement » au sens où, à partir de l'émanation première encore indéterminée qui vient de l'Un, ils se co-déterminent et se donnent l'un à l'autre limite et consistance. En ce sens, on pourrait tisser une continuité entre la sunhupostasis plotinienne et l'authupostaton de Proclus, que l'on traduit habituellement par « auto-constitution » mais qui signifie plus littéralement la manière dont toute ousia intelligible se fait exister dynamiquement elle-même.

En outre, S. Roux repère, dans le Traité 39, un transfert de ce modèle de la coexistence au niveau de l'Un : il désigne désormais l'articulation entre la quasi-réalité du premier principe et sa liberté ou sa volonté et permet de penser l'auto-production 
originelle du principe. L'analyse que propose l'auteur de l'efficacité de ce modèle pour penser l'existence de l'Un est très originale et convaincante. Elle doit toutefois être complétée par le rappel d'un scrupule ultime qui saisit Plotin dans les derniers chapitres du traité : le modèle de la coexistence se trouve désormais délaissé, puisque l'Un apparaît alors « seul, vraiment libre (...) alors que chacun des autres êtres est à la fois lui-même et autre chose » (21,31-33). Au modèle de la coexistence il faut désormais préférer la position d'une pure solitude qui coïncide avec la liberté première. La métaphysique de l'Un ne saurait s'en tenir à subvertir le paradigme substratif de l'ontologie d'Aristote : elle instaure un nouveau régime de la métaphysique qui rompt radicalement avec tout modèle d'articulation interne de l'être, qu'il s'agisse du substrat, de l'inclusion réciproque ou de la coexistence.

LAURENT LAVAUD

Université de Paris 1-PAnthéon Sorbonne/UMR 7219

\section{A. Longo \& D. P. Taormina (éd.), Plotinus and Epicurus. Matter, Perception,} Pleasure, Cambridge, Cambridge University Press, 2016, 236 p., ISBN : 978-1-10712421-9.

Des grands courants philosophiques de l'époque hellénistique que Plotin reprend et discute, l'épicurisme est, pour ainsi dire, le parent pauvre. Aussi pendant longtemps, la littérature secondaire avait-elle pratiquement négligé l'étude de sa réception dans les Ennéades. En s'emparant de cette question, cet ouvrage collectif fait bien davantage que pallier une lacune scolastique. Il s'inscrit dans une perspective ouverte récemment visant à mettre en lumière les points sur lesquels l'épistémologie plotinienne reprend et adapte certains éléments issus de l'épicurisme. Elle s'était développée il y a une dizaine d'années, à partir d'une discussion autour du statut de l'ennoia dans le traité 45 (III, 7) : inversant la lecture traditionnelle selon laquelle toute ennoia serait la trace dans l'âme des Formes transcendantes, B. van den Berg (« As we are always speaking of them and using their names on every occasion ... », dans R. Chiaradonna \& F. Trabattoni (éd.), Physics and Philosophy of Nature in Greek Neoplatonism, Leiden, 2009) soutenait que, selon Plotin, l'ennoia du temps aurait, à la différence de l'ennoia de l'éternité, une origine empirique. P. Remes (« Plotinus on Starting Points of Reasoning », Chôra 14, 2016) reprenant cette question, avait ensuite examiné plus spécialement la place que Plotin confere à certaines conceptions, formées à partir de l'expérience, qui, sans être des critères du vrai, ne sont pas non plus dépourvues de toute fiabilité, puisqu'elles peuvent servir de points de départ à la réflexion et à l'analyse philosophiques. Ce volume, consacré entièrement aux multiples aspects du rapport que Plotin entretient avec Épicure et l'épicurisme, approfondit considérablement les recherches dans cette voie. Mais ce n'est là qu'un aspect de la question. Plusieurs de ses contributions proposent de lire certains passages des Ennéades, tels que Enn. V, 5 (32), 1 ou Enn. III, 2 (47), 7, comme des éléments repérables du dossier anti-épicurien, révélant l'existence d'un véritable dialogue avec l'épicurisme dans les traités. Enfin et surtout, il permet d'aller bien au-delà des pistes ouvertes en 1981 par J.-P. Dumont (« Plotin et la doxographie épicurienne », dans Néoplatonisme, Mélanges offerts à Jean Trouillard, 1981) qui, s’intéressant aux allusions à l'atomisme dans les traités, restreignait la connaissance que Plotin avait de l'épicurisme aux simples compilations doxographiques.

Le recueil comporte, outre l'introduction, dix contributions, réparties en quatre sections de taille inégale. Entre la première, qui est un aperçu historique extrêmement bien documenté de T. Dorandi concernant l'état de la diffusion des écrits d'Épicure à 